\title{
Generación de un protocolo para la síntesis de nanopartículas de quitosano cargadas con florfenicol a través del método de gelación iónica
}

\author{
Generation of a protocol for the synthesis of chitosan nanoparticles loaded with \\ florfenicol through the ionic gelation method
}

Erico R. Carmona ${ }^{3}$, Tania Plaza ${ }^{3}$, Gonzalo Recio-Sánchez ${ }^{2,3}$, Jorge Parodi ${ }^{1,4}$

\section{Resumen}

\begin{abstract}
La formación de nanopartículas de quitosano mediante el método de gelación iónica tiene un alto potencial para la carga, transporte y liberación controlada de moléculas. Es un método sencillo y las nanopartículas presentan buena absorción, estabilidad, biocompatibilidad y baja toxicidad. El florfenicol es uno de los antibióticos más utilizados en la industria acuícola y es administrado mezclado con el alimento en altas concentraciones, lo cual genera problemas de dosis efectivas y de residuos no deseados. De esta manera, la síntesis de nanopartículas de quitosano (Q) cargadas con florfenicol podría otorgar ventajas a la hora de proteger, transportar y liberar de forma controlada el fármaco en peces de interés económico. En el presente estudio se sintetizaron nanopartículas de quitosano y tripolifosfato de sodio (TPP) con y sin florfenicol para evaluar su carga efectiva y liberación. Los resultados obtenidos con espectrometría FTIR corroboran la formación de las nanopartículas de Q-TPP con los cambios observados en los patrones de espectros infrarojo entre el quitosano matriz y las nanopartículas sintetizadas. Las nanopartículas de Q-TPP pueden cargar entre 48 y 50\% de florfenicol en su interior. El ensayo de liberación in vitro del antibiótico mostró una liberación controlada y estable en el tiempo en condiciones de $\mathrm{pH}$ ácido y temperatura controlada de $15^{\circ} \mathrm{C}$ de hasta 10 días. Finalmente, se muestra que las nanopartículas de Q-TPP tienen potencial para atrapar y liberar de forma controlada el fármaco evaluado.
\end{abstract}

Palabras clave: antibiótico; liberación de drogas; polímeros; nanomateriales

${ }^{1}$ Laboratorio de Biología Celular y Molecular Aplicada, Facultad de Ciencias, Vicerrectoría de Investigación y Postgrado, Universidad Mayor, Temuco, Chile

${ }^{2}$ Departamento de Ciencias Matemáticas y Físicas, Facultad de Ingeniería, Universidad Católica de Tетисо, Tетисо, Chile

${ }^{3}$ Núcleo de Investigación en Bioproductos y Materiales Avanzados (BioMA), Facultad de Ingeniería, Universidad Católica de Temuco, Temuco, Chile

${ }^{4}$ E-mail: jorge.parodi@umayor.cl

Recibido: 4 de abril de 2018

Aceptado para publicación: 13 de abril de 2018 
The formation of nanoparticles of chitosan by the ionic gelation method has a high potential for loading, transport and controlled release of molecules. It is a simple method and the nanoparticles have good absorption, stability, biocompatibility and low toxicity. Florfenicol is one of the most used antibiotics in the aquaculture industry and is administered mixed with the feed in high concentrations, which generates problems of effective doses and unwanted residues. In this way, the synthesis of chitosan (Q) nanoparticles loaded with florfenicol could provide advantages when it comes to protecting, transporting and releasing the drug in fish of economic interest in a controlled manner. In the present study, nanoparticles of chitosan and sodium tripolyphosphate (TPP) with and without florfenicol were synthesized to evaluate their effective loading and release. The results obtained with FTIR spectrometry corroborate the formation of the Q-TPP nanoparticles with the changes observed in the infrared spectra patterns between the matrix chitosan and the synthesized nanoparticles. Q-TPP nanoparticles can carry between 48 and $50 \%$ of florfenicol inside. The in vitro release assay of the antibiotic showed a controlled and stable release over time under conditions of acid $\mathrm{pH}$ and controlled temperature of $15{ }^{\circ} \mathrm{C}$ for up to 10 days. Finally, it is shown that the Q-TPP nanoparticles have the potential to trap and release the evaluated drug in a controlled manner.

Key words: antibiotics; drug release; polymer; nanomaterials

\section{INTRODUCCIÓN}

El quitosano (Q) es un polímero biodegradable, biocompatible, semipermeable y con propiedades antimicrobianas, lo cual lo convierte en un material versátil para múltiples aplicaciones (Kumar et al. 2000; Hamed et al., 2016). Este polímero ha venido recibiendo mucha atención debido a sus propiedades de adsorción y encapsulamiento, lo que permite empaquetar o cargar diferentes tipos de moléculas. De esta manera, el quitosano viene siendo sintetizado en diferentes tipos de materiales avanzados, tales como microesferas, hidrogeles, películas, micro y nanopartículas (Agnihotri et al., 2004; Sinha et al., 2004; Cafaggi et al., 2007).

Las partículas basadas en quitosano han sido extensivamente estudiadas para la liberación de drogas anticancerígenas, hormonas, genes, antígenos y antibióticos, entre otros (Gan et al., 2005; Derakhshandeh et al.,
2012; Mohammadpour et al., 2012; Mukhopadhyay et al., 2013; Ibrahim et al., 2015). Es importante mencionar que las nanopartículas de quitosano son partículas sólidas coloidales con un tamaño entre $1 \mathrm{y}$ $1000 \mathrm{~nm}$, con propiedades fisicoquímicas interesantes en relación a tamaño, estabilidad, adsorción, alta reactividad y facilidad de alcanzar los tejidos diana. Este tipo de nanopartículas es más ventajoso a la hora de cargar, transportar y liberar fármacos en comparación a materiales de escala micrométrica.

Para la preparación de nanopartículas de quitosano existen metodologías tales como coprecipitación, reticulación química, termodescomposición, coacervación, emulsificación y gelación iónica. Este último método es mayormente usado para la preparación de nanopartículas de polisacáridos (Raveendran et al., 2015). La técnica está basada en las interacciones iónicas entre los grupos aminos cargados positivamente del quitosano y los grupos cargados negativamen- 
te de un polianión, como por ejemplo el tripolifosfato de sodio (TPP), el cual es el agente iónico reticulador más ampliamente usado, debido a que no es tóxico y tiene la capacidad de gelificar rápidamente (Calvo et al., 1997; De Moura et al., 2009). De esta manera, las nanoparticulas Q-TPP son de gran atractivo, ya que son promisorios transportadores para la entrega de macromoléculas (Nagpal et al., 2010).

Entre los agentes antimicrobianos de uso veterinario más utilizados en la industria acuícola se encuentra el florfenicol (Burridge et al., 2010). Su mecanismo de acción es la inhibición de la síntesis de proteínas, a través de la unión a la subunidad 50S del ribosoma bacteriano, previniendo la transferencia de los aminoácidos desde el tRNA hacia la cadena peptídica en crecimiento, inhibiendo así la actividad peptidil transferasa (Schwarz et al., 2004). Este antibiótico es principalmente utilizado en mezcla con el alimento para infecciones ocasionados por Piscirickettsiosis y Renibacteriosis (Hayes, 2005). Sin embargo, gran parte del antibiótico administrado se pierde en el proceso de alimentación de los peces, lo cual genera pérdidas del fármaco previas a su consumo y la liberación de residuos en el medio ambiente acuático. De esta manera, nuevos métodos de encapsulación estables que protejan y liberen el antibiótico de forma controlada en peces de interés económico son necesarios a la hora de resolver este tipo de problemáticas para la industria acuícola.

Los objetivos del presente trabajo fueron: 1) sintetizar nanopartículas de Q-TPP a través del método de gelación iónica con TPP, 2) evaluar la capacidad y efectividad de carga o encapsulado del antibiótico florfenicol en nanopartículas de Q-TPP y, 3) evaluar la liberación in vitro del fármaco en condiciones fisiológicas similares a peces modelo de la industria acuícola.

\section{Materiales y MéTodos}

\section{Reactivos}

El polímero quitosano $(\mathrm{Q})$ de bajo peso molecular con grado desacetilación de 75$85 \%$ y el tripolifosfato de sodio (TPP) fueron adquiridos de Sigma-Aldrich (EEUU), el florfenicol (50\%) (FF) de Centrovet (Chile) y el ácido acético glaciar $100 \%$ de Merck (Alemania).

\section{Nanopartículas}

Las nanopartículas de quitosano se prepararon mediante el método de gelación iónica de acuerdo con las metodologías propuestas por Calvo et al. (1997) con algunas modificaciones. El quitosano de bajo peso molecular fue disuelto en tres soluciones acuosas de ácido acético glacial $(0.5,1.0$ y $1.5 \% \mathrm{v} / \mathrm{v})$ para formar una solución de concentración final de $1.25 \mathrm{mg} / \mathrm{ml}$. Esta solución se mantuvo en agitación por $24 \mathrm{~h}$ a temperatura ambiente utilizando un agitador magnético. $\mathrm{El} \mathrm{pH}$ inicial resultante fue de 3.5 , pero fue ajustado a 4.7 usando una solución de $\mathrm{NaOH} 1 \mathrm{~N}$. La solución fue filtrada a través de una jeringa con un tamaño de poro de $0.45 \mu \mathrm{m}$ para remover residuos de partículas insolubles. El TPP fue preparado y disuelto en agua ultrapura a una concentración final de $0.56 \mathrm{mg} / \mathrm{ml}$ con ayuda de agitación magnética.

El TPP fue agregado gota a gota con una jeringa $2 \mathrm{ml}$ en la solución de $5 \mathrm{ml} \mathrm{de}$ quitosano para la elaboración de las nanopartículas de Q-TPP. La mezcla se mantuvo en constante agitación durante $30 \mathrm{~min}$ a temperatura ambiente (Figura 1a). Luego, la suspensión resultante con las nanopartículas (Figura 1b) se centrifugó a $3200 \mathrm{rpm}$ por 20 min. Los pellets obtenidos fueron aislados del sobrenadante (Figura 1c), congelados a $-20^{\circ} \mathrm{C}$ y secados por $24 \mathrm{~h}$ usando un equipo liofilizador Virtis Bench Top K (Figura 1d). 
Para determinar la formación de nanopartículas de Q-TPP se realizó un análisis de espectro infrarrojo de las muestras obtenidas mediante el método de gelación iónica. El análisis FTIR se llevó a cabo utilizando un espectrómetro FTIR PerkinElmer (FTIR System Spectrum BX). Las muestras se prepararon usando la técnica de pastillas con bromuro de potasio $(\mathrm{KBr})$ y se analizaron para comprobar la presencia de nanopartículas sintetizadas en comparación a la muestra matriz de quitosano puro. Los espectros FTIR se recogieron en una resolución espacial de $4 \mathrm{~cm}^{-1}$ en el modo de transmitancia, entre $4000-450 \mathrm{~cm}^{-1}$, respectivamente.

Se agregó gota a gota una solución de 2 $\mathrm{ml}$ de TPP a $5 \mathrm{ml}$ de quitosano conteniendo 2 $\mathrm{mg} / \mathrm{ml}$ de florfenicol. La solución se mantuvo en agitación magnética durante $30 \mathrm{~min}$. Las nanopartículas resultantes en la suspensión se centrifugaron a $900 \mathrm{~g}$ durante $20 \mathrm{~min}$. Todos los pellets obtenidos se conservaron por $24 \mathrm{~h}$ a $-20{ }^{\circ} \mathrm{C}$ para posteriormente ser liofilizados por $48 \mathrm{~h}$.

\section{Eficiciencia de Carga}

La eficiencia de encapsulación y la capacidad de carga de las nanoparticulas de Q fue determinada por la separación de las nanoparticulas formadas desde el medio acuoso por centrifugación a $900 \mathrm{~g}$ durante $30 \mathrm{~min}$. La cantidad libre de florfenicol en el sobrenadante fue medido por espectrofotometría UV-Vis usando un espectrofotómetro Shimadzu UV-mini 1240, a $265 \mathrm{~nm}$ (Elimam et al., 2017). E1 florefenicol cargado en las nanopartículas de Q-TPP fue calculado según la siguiente formula (Saha et al., 2010): Porcentaje de carga $(\%)=(\mathrm{Ta}-\mathrm{Tal}) *(100 / \mathrm{Ta})$, donde Ta corresponde al florfenicol total usado para preparar las nanopartículas y Tal es el florfenicol libre en el sobrenadante.

\section{Liberación de Florfenicol}

Las nanopartículas cargadas con el antibiótico florfenicol previamente liofilizadas (1 $\mathrm{mg}$ ) fueron dispersadas en $10 \mathrm{ml}$ de solución salina de fosfato PBS (Life Technology Corporation, EEUU) a pH 3 y $\mathrm{pH}$ 7.4. Las supensiones fueron mantenidas en agitación continúa a $15^{\circ} \mathrm{C}$ con un agitador magnético. El espectro UV-Vis del florfenicol libre en el medio fue monitoreado a $265 \mathrm{~nm}$ en intervalos predeterminados de tiempo $(30,60,90$ min, y luego 12, 24, 72, hasta 264 h).

\section{Resultados y Discusión}

\section{Preparación de Nanopartículas Q-TPP}

Diferentes protocolos de dilución del quitosano con ácido acético $(0.5 \%-1.5 \% \mathrm{v} / \mathrm{v})$, concentraciones de quitosano $(0.5-1.5 \mathrm{mg} / \mathrm{ml})$,

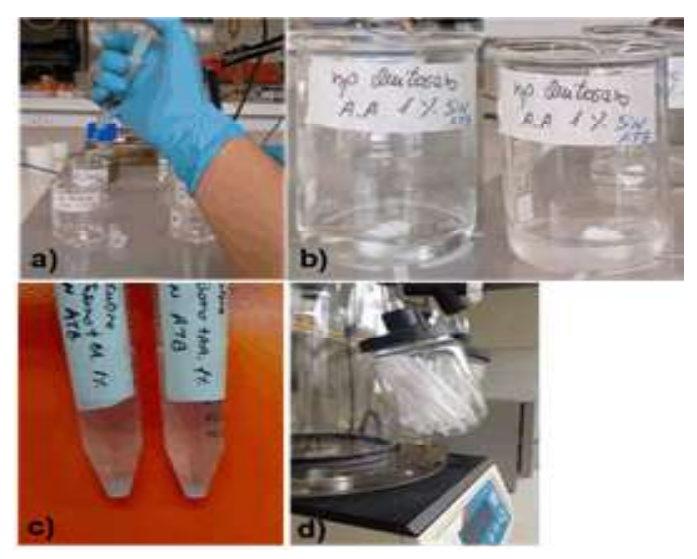

Figura 1. Preparación de nanopartículas de quitosano a través del método gelación iónica. a) adición de TPP a una solución de quitosano, b) formación espontánea de nanopartículas de Q-TPP (solución de color opalescente), c) centrifugación de nanopartículas en suspensión y formación de pellets conteniendo nanopartículas (botón de color opalescente), d) liofilización de nanopartículas 


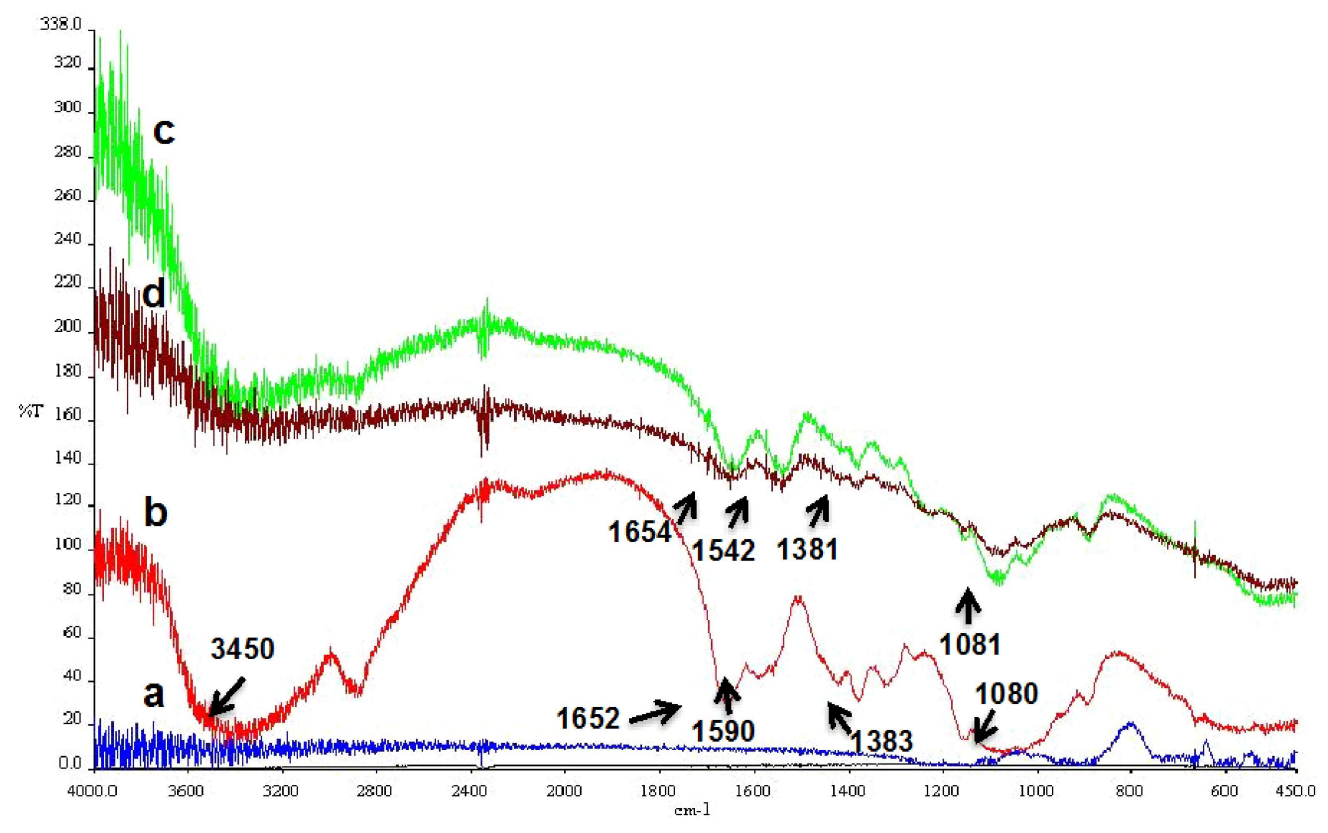

Figura 2. Espectro FTIR de nanopartículas de Q-TPP con el método de gelación iónica (porcentaje de la transmitancia, \%T). a) espectro del tripolifosfato de sodio (TPP); b) quitosano; c) nanopartículas de Q-TPP; d) nanopartículas Q-TPP cargadas con florfenicol

concentraciones de TPP $(0.3-1.0 \mathrm{mg} / \mathrm{ml})$ y con y sin la aplicación de ultrasonido fueron probadas previamente para formar nanopartículas de Q-TPP (resultados no mostrados). Los mejores resultados fueron obtenidos con una dilución del quitosano en ácido acético entre 1.0 y $1.5 \% \mathrm{v} / \mathrm{v}$, el uso de una concentración de $1.25 \mathrm{mg} / \mathrm{ml}$ de quitosano, TPP a una concentración de $0.56 \mathrm{mg} / \mathrm{ml}$ y sin la aplicación de ultrasonido. Lo anterior, fue evidenciado por la formación homogénea de nanopartículas de color opalescente y sin aglomerados en la solución final (Figura 1b) y los análisis de espectros infrarrojo de las muestras.

El análisis de los espectros FTIR de las partículas liofilizadas de Q-TPP confirmó la síntesis de nanopartículas de Q-TPP. En el espectro FTIR del quitosano (Figura 2b) se observaron los picos característicos de la molécula. A los $3450 \mathrm{~cm}^{-1}$ son correspondien- tes a la vibraciones de los grupos funcionales $\mathrm{OH}$ (Haider et al,. 2017). A los $1652 \mathrm{~cm}^{-1}$ y a los $1590 \mathrm{~cm}^{-1}$ aparecen los picos con alta intensidad de transmitancia característicos del quitosano, los cuales corresponden a las vibraciones de las bandas amida I y amida II, respectivamente (Papadimitriou et al., 2008). A los $1383 \mathrm{~cm}^{-1}$ se observó el tramo de los enlaces C-N, y a los $1081 \mathrm{~cm}^{-1}$ se observó el pico correspondiente al tramo $\mathrm{C}-\mathrm{O}$.

En la Figura 2 se muestra el espectro FTIR de nanopartículas de Q-TPP con el método de gelación iónica. Los espectros de estas partículas con o sin antibiótico se muestran diferentes a la de quitosano matriz puro. El ensanchamiento de la banda correspondiente a los grupos $\mathrm{OH}\left(3450 \mathrm{~cm}^{-1}\right)$ indica probablemente la formación de enlaces hidrógeno entre los oxígenos del tripolifosfato y los hidrógenos de los grupos amino del quitosano. Por otro lado, la transmitancia de 


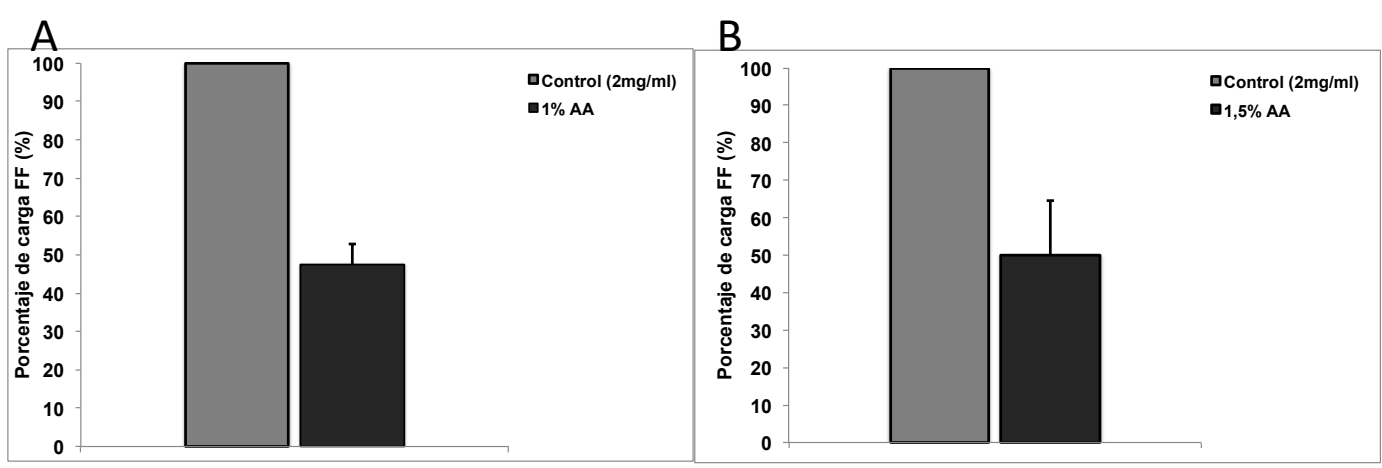

Figura 3. Porcentaje de carga de nanopartículas de Q-TPP con florfenicol. Nanopartículas preparadas con $1 \%$ (a) y $1.5 \%$ (b) de ácido acético glacial

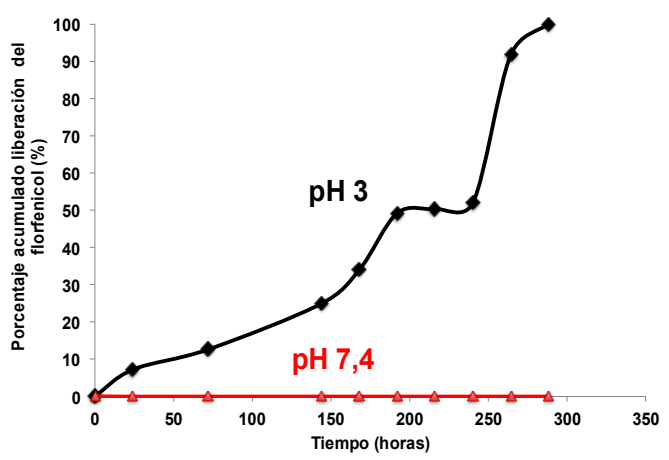

Figura 4. Liberación in vitro del antibiótico florfenicol desde nanopartículas de Q-TPP en medio salino PBS con $\mathrm{pH}$ 3 y 7 a $15^{\circ} \mathrm{C}$

\section{Nanopartículas de Q-TPP con Florfenicol}

La capacidad y eficiencia de carga de las nanopartículas sintetizadas fue levemente mayor para las nanopartículas preparadas usando $1.5 \%$ de ácido acético en comparación a las preparadas con un $1 \%(50.1 \mathrm{y}$ $48.0 \%$, respectivamente) (Figura 3). Esto se debe a que una concentración mayor de ácido acético mejoraría la solubilidad de los polímeros de quitosano y aumentaría, a su vez, el grado de protonación del quitosano, favoreciendo la capacidad potencial de formar entrecruzamientos con el anión TPP, promoviendo un mayor encapsulamiento del fármaco (Fan et al., 2012).

\section{Liberación in vitro del Florfenicol}

Los datos obtenidos de la liberación in los grupos amino se desplazan a 1654 y 1542 $\mathrm{cm}^{-1}$, lo que indica que estos grupos funcionales interactuaron con el TPP creando enlaces iónicos. Estas interacciones reducen la solubilidad quitosano y son responsables de la separación del quitosano desde la solución en forma de nanopartículas y la cual puede visualizarse con un cambio de color transparente hacia un color opalescente característico (Papadimitriou et al., 2008). Finalmente, un desplazamiento de las bandas $1381 \mathrm{~cm}^{-1} \mathrm{y}$ $1081 \mathrm{~cm}^{-1}$ respecto al quitosano matriz fue observado para las nanopartículas, lo que indica interacciones de los iones de TPP con grupos C-N y C-O, respectivamente. vitro del antibiótico florfenicol de las nanopartículas de Q-TPP en medio salino PBS a dos pH (neutro 7.4 y ácido 3.0) y a una temperatura de $15^{\circ} \mathrm{C}$ se muestran en la Figura 4. Esta situación simula las condiciones fisiológicas de un pez modelo. La liberación comienza recién a las $24 \mathrm{~h}$ de incubación en el medio ácido, mientras que la liberación del 50\% del antibiótico comienza pasadas las 216 h (9 días), culminando a las 264 h (11 días). Los resultados muestran una liberación controlada y prolongada del fármaco bajo estas condiciones experimentales. Estos resultados difieren notablemente a los docu- 
mentados por otros autores que muestran una liberación restringida de diferentes tipos de antibióticos entre 24 y 72 h (Saha et al., 2010; Zhao et al., 2013; Huang et al., 2014); sin embargo, dichos estudios fueron realizados con un rango de temperaturas más altas $\left(25-37{ }^{\circ} \mathrm{C}\right)$ que la usada en el presente estudio, demostrando que la temperatura es un factor relevante en el proceso de liberación de drogas.

En el ensayo con $\mathrm{pH}$ neutro no se observó liberación del antibiótico durante el periodo total de la prueba (11 días). Lo anterior podría ser debido a la buena estabilidad de las nanopartículas sintetizadas de Q-TPP bajo la metodología descrita en este trabajo.

\section{Conclusiones}

- La técnica de gelación iónica es una metodología útil para sintetizar nanopartículas de quitosano y tripolifosfato de sodio (TPP).

- La concentración del quitosano, ácido acético y TPP son factores de importancia para formar nanopartículas cargadas con el antibiótico florfenicol.

- Las nanopartículas fabricadas mostraron una buena estabilidad, capacidad de carga y una liberación controlada del florfenicol, demostrando que las nanopartículas de Q-TPP tienen potencial para atrapar y liberar de forma controlada este tipo de antibiótico.

\section{Agradecimientos}

FONDECYT 11150322, proyecto Grupo de Investigación Bio-Nanomateriales, Universidad Católica de Temuco N. ${ }^{\circ}$ 201GICI-01, Proyecto OI 101340 de la Universidad Mayor.

\section{Literatura Citada}

1. Agnihotri SA, Mallikarjuna NN, Aminabhavi TM. 2004. Recent advances on chitosan-based micro-and nanoparticles in drugs delivery. J Control Release 100: 5-28. doi: 10.1016/ j.jconrel.2004.08.010

2. Burridge L, Weis JS, Cabello F, Pizarro J, Bostick K. 2010. Chemical use in salmon aquaculture: a review of current practices and possible environmental effects. Aquaculture 306: 7-23. doi: 10.1016/j.aquaculture.2010.05.020

3. Cafaggi S, Russo E, Stefani R, Leardi $R$, Caviglioli G, Parodi B, Bignardi $G$, et al. 2007. Preparation and evaluation of nanoparticles made of chitosan or Ntrimethyl chitosan and a cisplatin-alginate complex. J Control Release 121: 110-123. doi: 10.1016/j.jconrel.2007.05.037

4. Calvo P, Remuñán-López C, Vila-Jato JL, Alonso MJ. 1997. Novel hydrophilic chitosan-polyethylene oxide nanoparticles as protein carriers. J Appl Polym Sci 63: 125-132. doi: 10.1002/ (S ICI) 1 097-4628(19970103) 63:1<125::AID-APP13>3.0.CO;2-4

5. Derakhshandeh K, Fathi S. 2012. Role of chitosan nanoparticles in the oral absorption of Gemcitabine. Int $\mathrm{J}$ Pharmaceut 437: 172-177. doi: 10.1016/ j.ijpharm.2012.08.008

6. de Moura MR, Aouada FA, AvenaBustillos RJ, McHugh TH, Krochta JM, Mattoso LHC. 2009. Improved barrier and mechanical properties of novel hydroxypropyl methylcellulose edible films with chitosan/tripolyphosphate nanoparticles. J Food Eng 92: 448-453. doi: 10.1016/j.jfoodeng.2008.12.015

7. Elimam MM, Shantier SW, Gadkariem EA, Mohamed MA, Osman $Z$. 2017. Stability studies on florfenicol using developed derivative spectrophotometric methods. Ann Pharm Fr 75: 40-44. doi: 10.1016/j.pharma.-2016.07.003

8. Haider J, Majeed H, Williams PA, Safdar W, Zhong F. 2017. Formation of chitosan nanoparticles to encapsulate krill oil (Euphausia superba) for application as a dietary supplement. Food Hydrocolloid 63: 27-34. doi: 10.1016/ j.foodhyd.2016.08.020 
9. Hamed I, Özogul F, Regenstein JM. 2016. Industrial applications of crustacean by-products (chitin, chitosan, and chitooligosaccharides): a review. Trends Food Sci Tech 48: 40-50. doi: 10.1016/j.tifs.2015.11.007

10. Hayes J. 2005. Determination of florfenicol in fish feed by liquid chromatography. J AOAC Int 88: 1777-1783.

11. Huang YC, Li RY. 2014. Preparation and characterization of antioxidant nanoparticles composed of chitosan and fucoidan for antibiotics delivery. Mar Drugs 12: 4379-4398. doi: 10.3390/ md12084379

12. Ibrahim HM, Manal KE, Ghada MT, Ekhlas AE. 2015. Chitosan nanoparticles loaded antibiotics as drug delivery biomaterial. J App Pharm Sci 5: 85-90. doi: 10.7324/JAPS.2015.501015

13. Kumar MN. 2000.0 A review of chitin and chitosan applications. React Funct Polym 46: 1-27. doi: 10.1016/S13815148(00)00038-9

14. Mohammadpour N, Eskandari R, Avadi MR, Zolfagharian H, Mir Mohammad A, Rezayat M. 2012. Preparation and in vitro characterization of chitosan nanoparticles containing Mesobuthus eupeus scorpion venom as an antigen delivery system. J Venom Anim Toxins 18: 44-52. doi: 10.1590/ S1678-91992012000100006

15. Mukhopadhyay P, Sarkar K, Chakraborty $M$, Bhattacharya $S$, Mishra R, Kundu PP. 2013. Oral insulin delivery by self-assembled chitosan nanoparticles: in vitro and in vivo studies in diabetic animal model. Mat Sci Eng C-Mater 33: 376-382. doi: 10.1016/ j.msec.2012.09.001

16. Nagpal K, Singh SK, Mishra DN. 2010. Chitosan nanoparticles: a promising system in novel drug delivery.
Chem Pharm Bull 58: 1423-1430. doi: 10.1248/cpb.58.1423

17. Papadimitriou S, Bikiaris D, Avgoustakis K, Karavas E, Georgarakis M. 2008. Chitosan nanoparticles loaded with dorzolamide and pramipexole. Carbohyd Polym 73: 44-54. doi: 10.1016/ j.carbpol.2007.11.007

18. Raveendran S, Palaninathan $V$, Nagaoka Y, Fukuda T, Iwai S, Higashi T, Mizuki T, et al. 2015. Extremophilic polysaccharide nanoparticles for cancer nanotherapy and evaluation of antioxidant properties. Int J Biol Macromol 76: 310-319. doi: 10.1016/j.ijbiomac.2015.03.001

19. Saha P, Goyal AK, Rath G. 2010. Formulation and evaluation of chitosanbased ampicillin trihydrate nanoparticles. Trop J Pharm Res 9: 483-488.

20. Sinha VR, Singla AK, Wadhawan S, Kaushik R, Kumria $R$, Bansal K, Dhawan S. 2004. Chitosan microspheres as a potential carrier for drugs. Int J Pharmaceut 274: 1-33. doi: 10.1016/ j.ijpharm.2003.12.026

21. Schwarz S, Kehrenberg C, Doublet B, Cloeckaert A. 2004. Molecular basis of bacterial resistance to chloramphenicol and florfenicol. FEMS Microbiol Rev 28: 519-542. doi: 10.1016/j.femsre.2004.04.001

22. Gan Q, Wang T, Cochrane $C$, McCarron P. 2005. Modulation of surface charge, particle size and morphological properties of chitosanTPP nanoparticles intended for gene delivery. Colloid Surface B 44: 65-73. doi: 10.1016/j.colsurfb.2005.06.001

23. Zhao L, Zhu B, Jia Y, Hou W, Su C. 2013. Preparation of biocompatible carboxymethyl chitosan nanoparticles for delivery of antibiotic drug. Biomed Res Int ID 236469. doi: 10.1155/2013/236469 